\title{
Ripensare i lastscapes. \\ Da spazi altri a spazi pubblici: ipertopie al di là dei recinti
}

\author{
Giovangiuseppe Vannelli \\ Dipartimento di Architettura, Università degli Studi di Napoli Federico II, Italia \\ giovangiuseppe.vannelli@unina.it
}

Angela D'Agostino

Dipartimento di Architettura, Università degli Studi di Napoli Federico II, Italia

angdagos@unina.it

\section{Rita Occhiuto}

Faculté d'Architecture, Université de Liège, Belgio r.occhiuto@uliege.be

\begin{abstract}
Nella geo-grafia di recinti che conforma la città in espansione novecentesca entrano in crisi processi di descrizione, interpretazione e progetto dello spazio pubblico. In questa complessa geografia, che spesso coincide con quella del drosscape, le eterotopie di deviazione pongono questioni spinose nel paesaggio urbano contemporaneo. È a partire dal recinto, elemento rappresentativo dell'essenza eterotopica di questi luoghi, che bisogna ripensare ruolo e conformazione di questi patrimoni ormai obsoleti.

II contributo indaga il paesaggio cimiteriale, i lastscapes, quale possibile occasione per i brani di città che li lambiscono. Questi luoghi vanno interpretati quali ipertopie e non più eterotopie, da spazi altri devono essere assunti quali spazi pubblici della città. Con questo scopo, avvalendosi di casi studio nordeuropei, si propongono tre strategie progettuali: un progetto di stanze, un progetto di margini, un progetto di layers.
\end{abstract}

\section{Parole chiave}

Eterotopie, cimiteri, publicness, memoria, paesaggio multifunzionale

\begin{abstract}
Conventional description, interpretation and design processes for public space enter into crisis in the geography of the 20th-century sprawling city denoted by enclosures. In this complex geography, often coinciding with that of drosscape, heterotopias of deviation raise thorny issues in the contemporary urban landscape. Role and shape of these now obsolete heritages must be rethought starting with the enclosure walls, a representative element of the heterotopical identity of these places.

This paper explores burial landscapes, lastscapes, as a chance for urban fragments surrounding them. These places should be interpreted as hypertopias and no longer as heterotopias, rather than "other spaces" they should be considered as public spaces. With this purpose, three design strategies are proposed, using northern European case studies: a project of rooms, a project of margins, a project of layers.
\end{abstract}

\section{Keywords}

Heterotopias, cemeteries, publicness, memory, multifunctional landscape 


\section{Una geo-grafia di recinti: \\ tra spazio pubblico e publicness}

II progetto d'architettura, nel confrontarsi con geografie urbane complesse che appaiono quali incoerenti estensioni delle morfologie delle città storiche, s'impone come uno strumento di rivelazione e di ricucitura di paesaggi frammentari fatti di scarti e di residui. Questi rimandano alla necessità di ridurre il consumo del suolo, restituendo valore e significato ai vuoti, composti da fragili brani di terra in attesa. A mutare è la percezione degli elementi conformativi della città: le relazioni spaziali tra pieni e vuoti, pubblico e privato, naturale e minerale vengono messe in crisi.

Ad una città storica densa, laddove i vuoti misurano le distanze tra le masse architettoniche o naturali che conformano la città, corrisponde una città in estensione che sovverte la prossemica tradizionale: non è più il pieno a conformare la città bensì i vuoti, le distanze, gli interstizi. L'emergenza di nuove 'enclave' e di frammenti dimenticati permette da un lato, di ricostruire reti e sistemi di nuova naturalità e dall'altro, di riscoprire il potenziale sistemico di tipologie come parchi, giardini, cimiteri e luoghi ibridi d'architettura e natura, concepiti come tasselli territoriali di finitezza aperta (Venturi Ferraiolo, 2001). II recinto diviene un dispositivo di privazione e privatizzazione, concausa della genesi degli spazi di scarto di cui le generiche periferie della città contemporanea sovrabbondano.
II recinto, temenos che perde il carattere eccezionale ed onorifico che gli era proprio presso i greci, diviene nel tempo moderno pura formalizzazione del verbo $\tau \varepsilon ́ \mu v \omega$, l'azione del tagliare.

Dunque, una geografia di recinti è il patrimonio urbano che ereditiamo dal Novecento, una 'geo-grafia tagliata': una scrittura sulla terra di muri che tracciano limiti. In questi brani di città, la preziosa possibilità, riscontrabile nei tessuti urbani consolidati di intendere lo spazio pubblico come una fonte inesauribile di sistemi spaziali e sociali costantemente ridefinibili, reinterpretabili e riconfigurabili sembra indebolita, o meglio ostacolata:

\begin{abstract}
'Recinto' è tutto ciò che costituisce il territorio attraverso la pura funzione di impedire l'attraversamento. Non necessariamente l'attraversamento di un corpo fisico, eventualmente quello dello sguardo, o di una legislazione. Questa definizione in termini di 'pura funzione' (ma altre funzioni spesso si sovrappongono) è quella che ci permette di sussumere sotto un'unica nozione oggetti apparentemente diversi: dal tripudio del dispotismo della Grande Muraglia alla modestia anonima, ma ancor più territorializzante, di un avviso che comunica al pubblico: 'White only' (Cerri et al., 1979, p. 7).
\end{abstract}

Questa relazione tra il recinto quale elemento adottato per conformare la città e lo spazio pubblico inteso come un cangiante sistema costantemente ri- 
tà non reinterpretati. Completamento e saturazione sono state le operazioni che hanno trovato spazio lungo le infrastrutture viarie che collegavano il centro città con queste cittadelle del lavoro, della salute, della morte. Ciò, anche in virtù dell'imperante logica dello zoning, ha contribuito alla strutturazione di un paesaggio incoerente e frammentato costituito di tessere di un mosaico non rispondenti ad uno stesso disegno complessivo e tenute insieme da uno spazio infrastrutturale che raramente ha assunto caratteri e caratteristiche tali da definire efficaci spazi pubblici.

Lo spazio pubblico detiene i caratteri per costruire sistemi complessi (Morin, 2000): questa conformazione urbana definita mediante un susseguirsi di recinti si può assumere come traccia evidente di un approccio che - parafrasando Morin - semplifica e unidimensionalizza la realtà, privandola di quegli intrecci che sono la radice etimologica, fenomenologica ed esperienziale della complessità.

Pensare e concepire luoghi interrelati tra di loro come parti elementari di un sistema complesso e globale, che deve la propria efficacia contemporaneamente al singolo elemento ed al tutto, è operazione quanto mai utile e necessaria per pensare allo spazio pubblico. Se nella città storica tale operazione si può ricon- te identificabili (piazze, portici, corti, ecc.) e variamente interpretabili, nella complessa geo-grafia di recinti è necessario re-inventare le logiche conformative per lo spazio pubblico e la sua stessa definizione. Lo spazio fisico va indagato in quanto attivatore di interazioni sociali, lo spazio interno ai recinti e il 'generico' spazio infrastrutturale che li lambisce vanno investigati nella loro capacità di costruire publicness, di costruire un paesaggio multifunzionale che vada oltre l'approccio mono-funzionalista egemone dello scorso secolo. Diversamente dallo spazio pubblico, tradizionalmente inteso quale luogo fisico preposto ad accogliere usi comuni, la publicness è un processo - talvolta determinato da azioni progettuali intangibili - una pratica collettiva che genera fenomeni di condivisione e scambio tra gli individui messi in scena in spazi inattesi e mutevoli, autopoieticamente assunti quali catalizzatori della vita pubblica. In questo contesto, il progetto deve accettare la sfida di leggere, introiettare e interpretare le differenti dinamiche urbane. II carattere composito, la pluridisciplinarietà e la temporalità differenziata delle strategie progettuali, ne fanno uno degli strumenti più complessi ed articolati capaci di adattarsi all'incertezza che caratterizza le mutazioni territoriali e sociali.

\section{Lastscapes:}

\section{obsolescenza tra memorie e nuove istanze}

Per osservare le geografie recenti, la lente del paesaggio fornisce un punto di vista utile a cogliere le relazioni deboli o dismesse che permeano questi brani di città. Si tratta di una operazione necessaria al fine di inserirsi nei o innescare dei processi di trasformazione rigenerando le connessioni interne al sistema diffuso degli spazi aperti così da reinterpretare la città-paesaggio determinando processi virtuosi di socialità e di cura degli spazi che abitiamo (Cortesi, Cappiello, 2017). La geo-grafia di recinti spesso risulta sovrapponibile alla "geografia del drosscape" (Bocchi, 2017, p. 21) assunta come una delle principali urgenze delle agende politiche. 

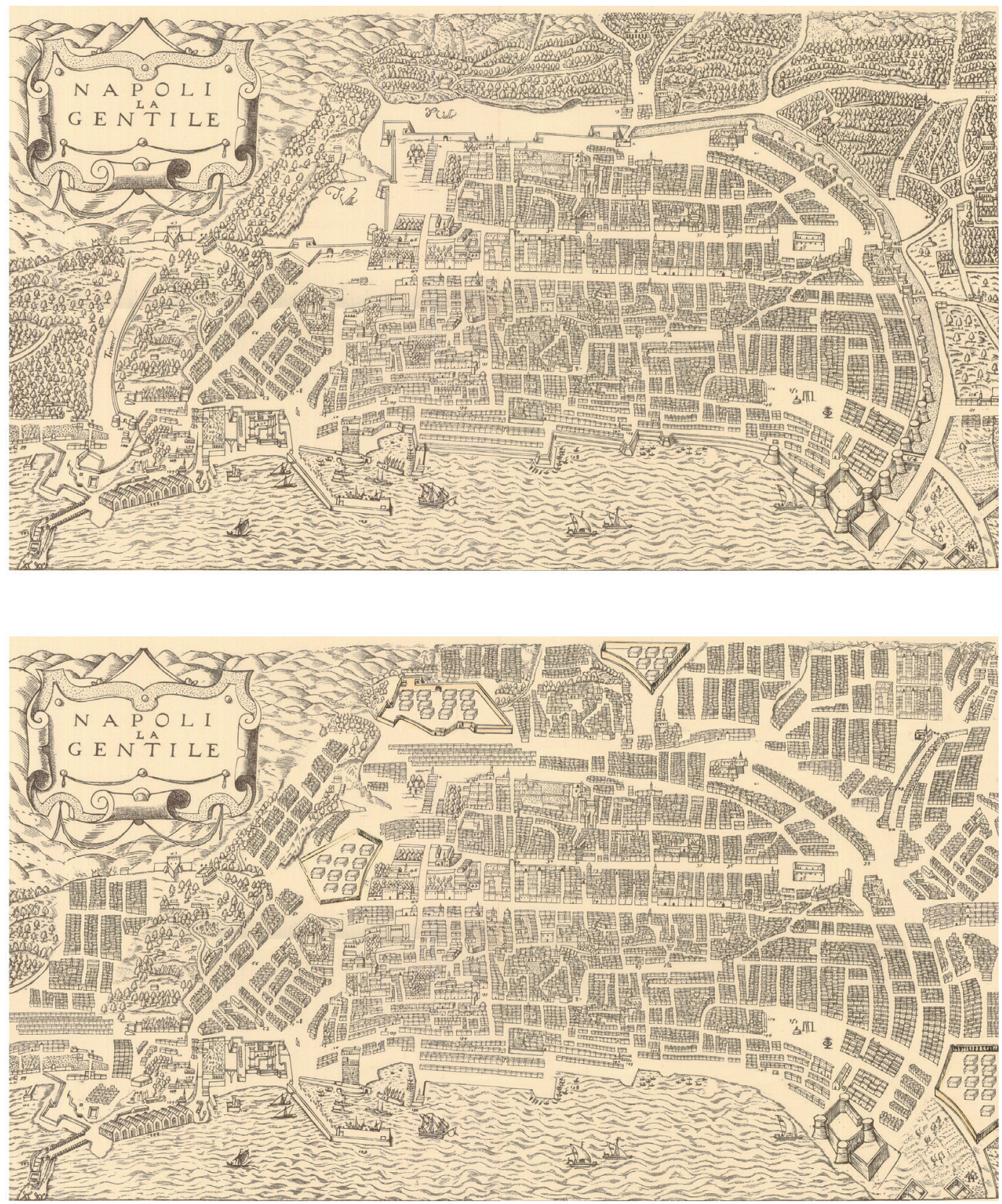

Fig. 1 - Manipolazione grafica della Cartografia ‘Napoli la Centile' di Nicolò Van Aelst, 1590. Trasformazioni urbane: dalle mura di cinta alle città recintate (elaborazione: G. Vannelli). 
Questa complessa geografia si conforma mediante l'inserimento di sistemi urbani autonomi e recintati che, utilizzando il dispositivo del recinto, 'tagliano' il paesaggio lasciandone frammenti residuali. Per comprendere la genesi di questi paesaggi di scarto è utile declinare in maniera specifica il concetto di obsolescenza (Abramson, 2016). I molteplici fenomeni di obsolescenza che hanno interessato queste cittadelle recintate hanno determinato un incrementale addensarsi di scarti, rifiuti e residui nei paesaggi ai margini della città densa: l'obsolescenza - fenomeno transcalare e complesso - assume forme differenti e richiede dunque azioni molteplici.

L'elemento del recinto interferisce notevolmente con questi processi di obsolescenza poiché la mancata percezione degli stessi può determinare un rallentamento o una accelerazione dei fenomeni in atto. Nei sistemi urbani recintati la presenza e la persistenza di un limes costruito che non si fa limen, collaborano alla dimenticanza e all'obsolescenza del 'contenuto' favorendone l'emarginazione, il rifiuto, l'esclusione. Per queste ragioni dunque, il recinto, oltre ad essere un dispositivo spaziale che rappresenta un regime proprietario, vincolistico, funzionale e anche un dispositivo d'ordine, nel corso del Novecento si è affermato quale elemento distintivo e connotante delle foucauItiane eterotopie di deviazione (Foucault, 2011), protagoniste indiscusse di questi brani di città.

Inoltre, particolarmente significativa è la dimensione intangibile di questi limiti che si manifesta nella distanza - emozionalmente percepita - che la società stabilisce rispetto a questi spazi. Anche per questa ragione, la condizione di obsolescenza in cui vertono gli spazi cimiteriali delle città medio-grandi è probabilmente la più complessa da interpretare e addirittura da vedere rispetto al fenomeno rilevabile presso le altre eterotopie come ospedali psichiatrici e carceri. Per ricercare ragioni, effetti e soluzioni possibili è necessario un approccio interdisciplinare: questo king lastscapes Perspectives ${ }^{2}$ a cui questo contributo fa riferimento.

Al fine di tracciare in maniera chiara la variabile relazione che intercorre tra eterotopia, obsolescenza e città per comprendere il potenziale ruolo di queste cittadelle nella definizione di nuovi spazi pubblici per la città di recinti, risulta necessaria una precisazione. Eterotopie di deviazione come ospedali psichiatrici e carceri sono concepite per ospitare la vita dei degenti o dei detenuti, il lavoro dei vari operatori e quel particolare rito della visita degli esterni che sostanzia l'essenza eterotopica di questi luoghi. Invece, i cimiteri sono realizzati al fine di dare una sistemazione presumibilmente eterna ai defunti, presenze intangibili che riempiono di valore e significato questi luoghi: "la particolare eterotopia del cimitero [...] è certamente un luogo altro nei confronti degli spazi culturali ordinari [...] tuttavia è solidale con l'insieme di tutti i luoghi della città, della società o del villaggio, ecc., poiché ogni individuo, ogni famiglia si trova ad avere dei parenti al cimitero" (Foucault, 2011, p. 26). Dunque, proprio in ragione di questa eccezionalità statutaria, il tema dello spazio pubblico risulta essere la chiave interpretativa portante per lo spazio eterotopico dedicato alla morte. Ciò che questi spazi sono chiamati a contenere è una assenza, una mancanza, un ricordo. Dunque, i veri abitanti - intesi come coloro i quali hanno l'abitudine - sono quelli che onorano queste memorie attraverso i riti, attraverso la presenza: i vivi. Una prima forma di obsolescenza si può dire sia semantica. II significato attribuito ai luoghi della sepoltura è mutato, per Masullo quello della sepoltura dei morti è considerato ormai come "un problema analogo all'eliminazione delle scorie industriali e dei rifiuti urbani" (Masullo, 2004, p. 19). Si è persa la continuità dei riti che precedentemente animava il camposanto: "con il trascorrere delle generazioni si allenta, fino a perdersi del tutto, tra i defunti e i vivi quella 'celeste corrispondenza di amorosi sensi' di foscoliana memoria" (Mangone,2004, pp. 27-28). 
Fino alla metà del secolo scorso, i cimiteri, sono stati luoghi di pellegrinaggio costante: luoghi di incontri domenicali e meta di stravaganti viaggiatori, i cimiteri sono stati a lungo percorsi, visitati, curati. Oggi invece: "death has no place in a society which is obsessed with youth and vigour" (Heathcote, 1999, p.11). Dunque, questi monumenti, in una pratica che dalla ri-conoscenza giunge alla re-invenzione, devono esser traghettati dal passato ad un possibile futuro. I lastscapes (De Leo, 2006) - crasi tra i termini last e landscape che sembra mettere in avanti l'idea di una compresenza ordinata di paesaggi della nostra vita (Morelli, 2020) - necessitano di una risemantizzazione poiché oggigiorno "esplodono per un'inarrestabile invasività, spoglia di qualsiasi funzione in favore dell'umanità, quindi sprovvista di qualsiasi valore" (Masullo, 2004, p. 19). A questa prima forma di obsolescenza strettamente relativa alla percezione e alla memoria se ne può far seguire una seconda che si potrebbe dire funzionale e prestazionale al contempo. Continua è la mutazione delle prassi che riguardano la sepoltura poiché da un lato vi sono sempre nuove tecniche e dall'altro le società divengono sempre più multireligiose. Questi due fenomeni necessitano che lo spazio dedicato alla sepoltura sia più complesso e capace di accogliere nuovi intrecci di molteplici e variabili istanze. Ciò significa non solo ripensare il patrimonio ereditato ma anche traghettare verso possibili evoluzioni i modelli della storia.

\section{Verso l'ipertopia: tre strategie progettuali}

Eterotopia, recinti, obsolescenza, memoria sono i principali termini che informano la spinosa questione del destino degli spazi cimiteriali: monumenti per eccellenza, monito e ricordo. Necessario è sdoganare quella "ossessione per la morte come malattia" (Foucault, 2011, p. 27) che Foucualt fa risalire al XIX secolo.

I lastscapes necessitano di essere reinterpretati nel loro ruolo urbano: da luoghi di reclusione devono divenire spazi dell'inclusione, da cittadelle per i morti a paesaggi per i vivi, da spazi altri a spazi pubblici. Ciò è necessario per contrastare quella 'pornografia della morte' di goreriana memoria: la morte ridotta a tabù, esiliata dalle conversazioni, una realtà da eludere. In questa direzione, l'azione progettuale tout-court è condizione necessaria ma non sufficiente, poiché diviene portante la cifra intangibile del progetto, qui intesa come azione di risignificazione e risemantizzazione che, anche mediante concrete azioni trasformative, ha quale obiettivo un'ampia riconsiderazione del valore e dei valori, in atto e in potenza, di questi luoghi.

Il progetto di architettura e di paesaggio per lo spazio cimiteriale, interpretando i vari contesti sociali e culturali, è chiamato a fornire risposte alle nuove istanze, alternative ai vecchi modelli e reinterpretazioni delle inedite relazioni contestuali. La molteplicità deve divenire paradigma. La unidimensionalizzazione della realtà prodotta dai processi di semplificazione su cui si è basata la costruzione della geo-grafia di recinti trova un'esemplare testimonianza nelle cittadelle eterotopiche. Eppure il paradigma della molteplicità non deve implicare la negazione dei caratteri eterotopici bensì una ricerca di complessità. II recinto non necessita di esser abbattuto, frantumato, negato, ma nella sua identitaria permanenza deve divenire inclusivo: il progetto deve tendere all'intreccio (complexus).

L'interpretazione proposta per questo patrimonio eterotopico, I'unico ad essere contraddistinto da una forza dell'inevitabile (Barberan, 2005), è presa a prestito dalla teoria cinematografica. L'eterotopia cimiteriale attraverso il progetto deve divenire "ipertopia": una nuova struttura spaziale in cui “non c'è più un aprirsi del 'qui' verso 'l'altrove' ma piuttosto un 'altrove' che arriva 'qui' e si scioglie in esso" (Casetti, 2015, p. 206). Dunque non è il recinto a dover essere rimosso per rendere fluida la continuità tra il 'qui' e 'I'altrove' bensì 'l'altrove' e il 'qui' devono instaurare un complesso rapporto di compresenza, un intreccio. 

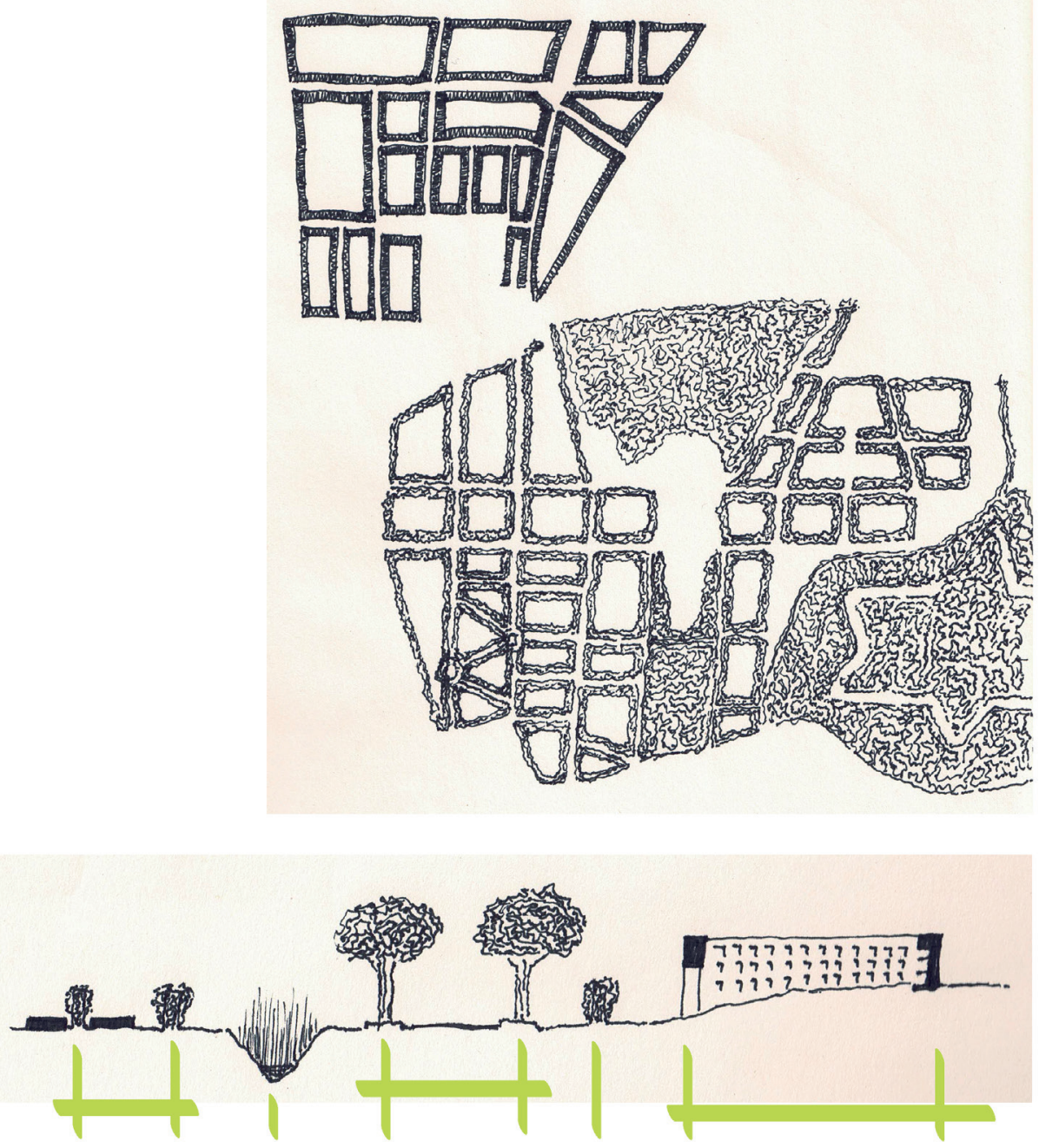

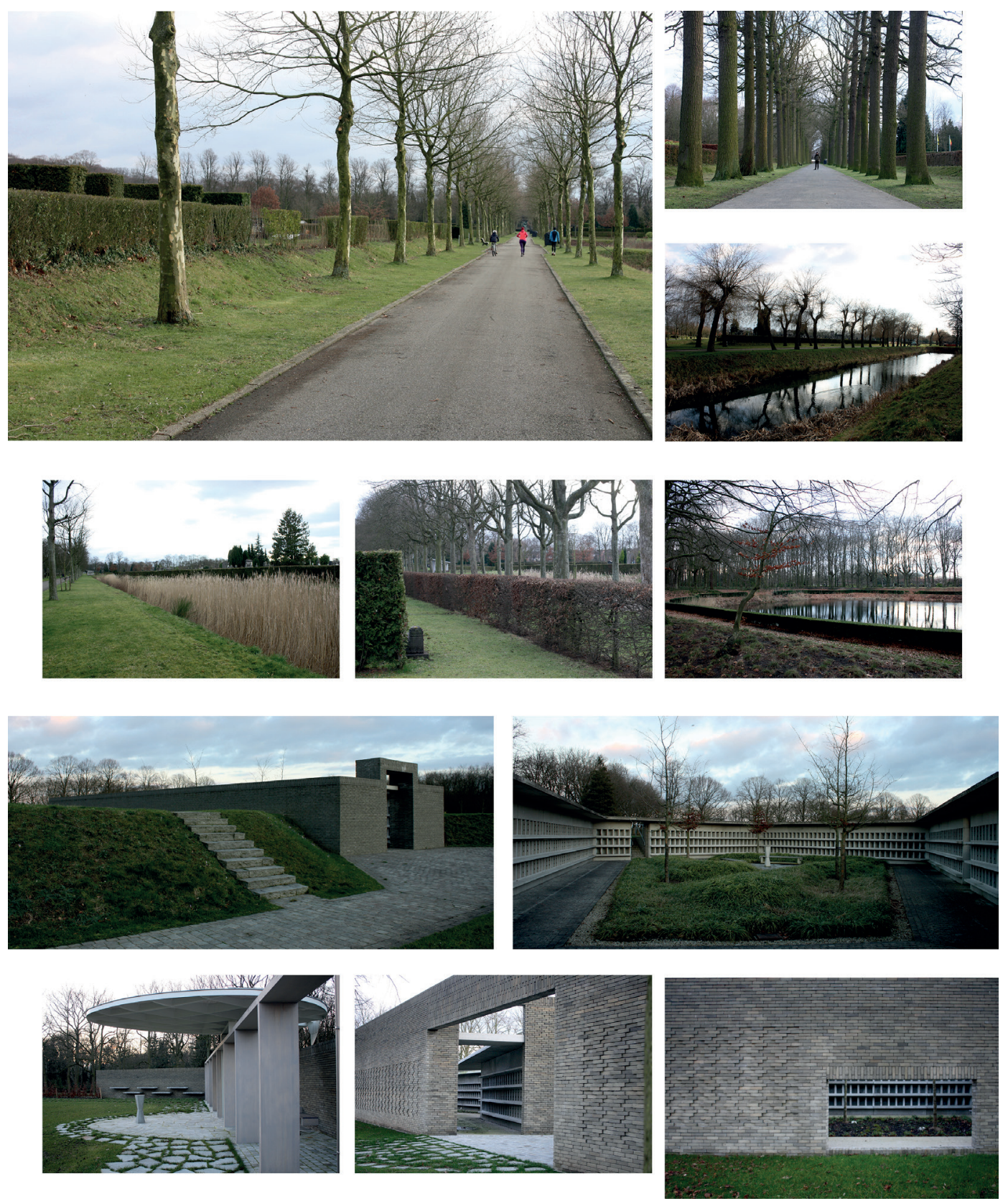

Fig. 3 - Schoonselhof Cemetery, Antwerpen, Belgio. Un progetto di stanze: successioni spaziali (foto: C. Vannelli). 

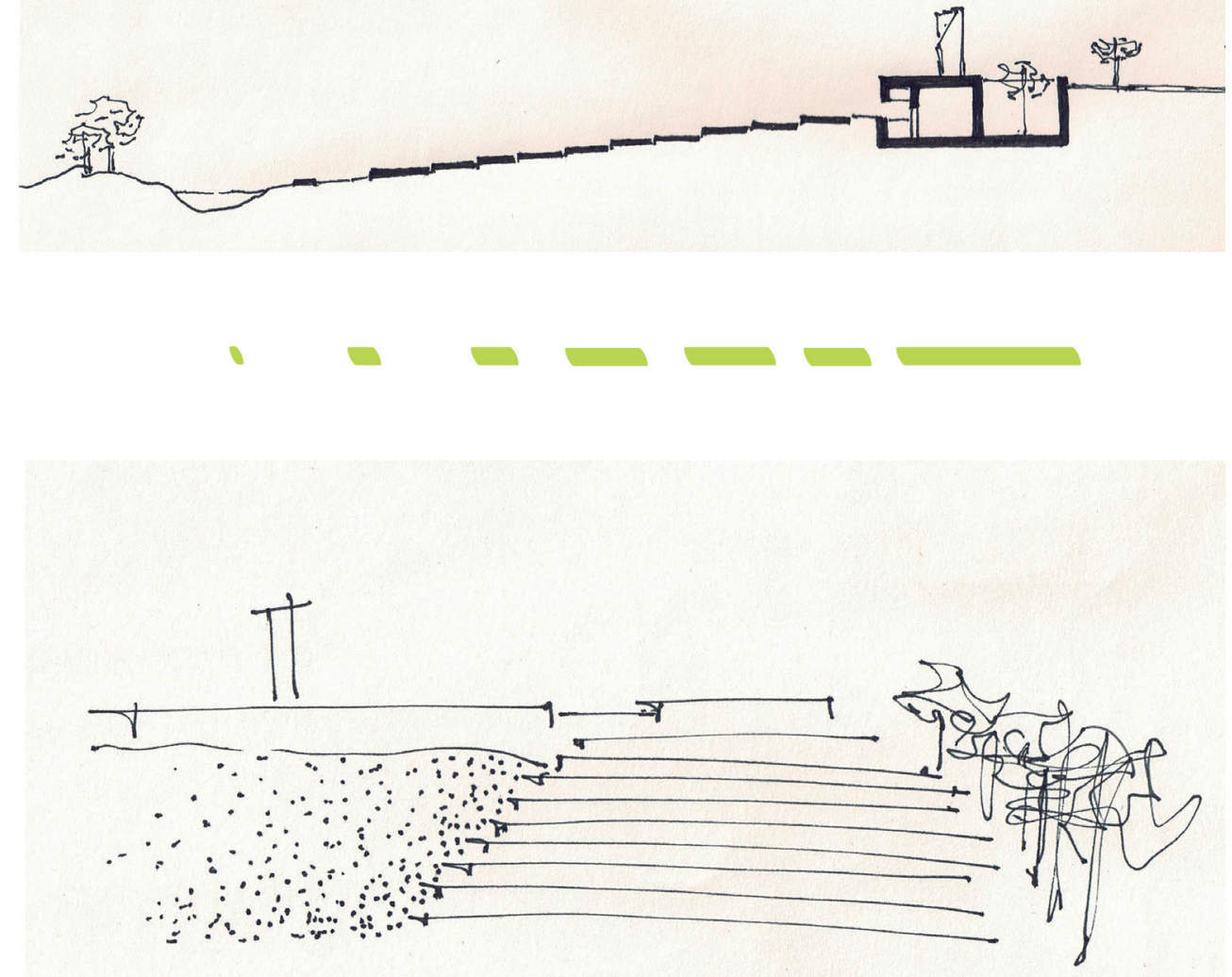

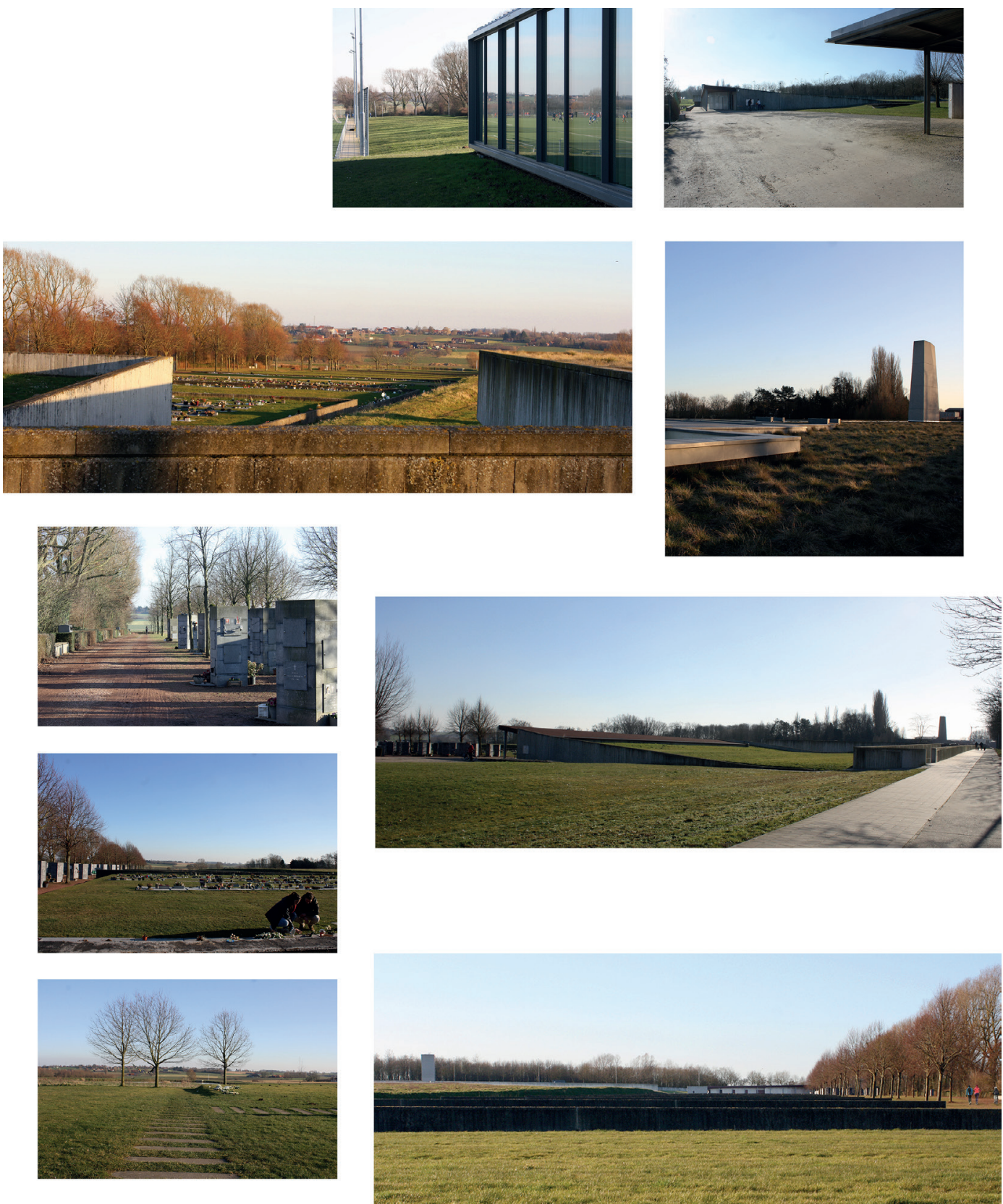

Fig. 5 - Begraafplaats Hoog Kortrijk, Kortrijk, Belgio. Un progetto di margini: la costruzione nel/del paesaggio (foto: G. Vannelli). 
$\longleftarrow$
$\bullet$
$\sim$
$\bullet$
$\bullet$
$\bullet$
$\bullet$
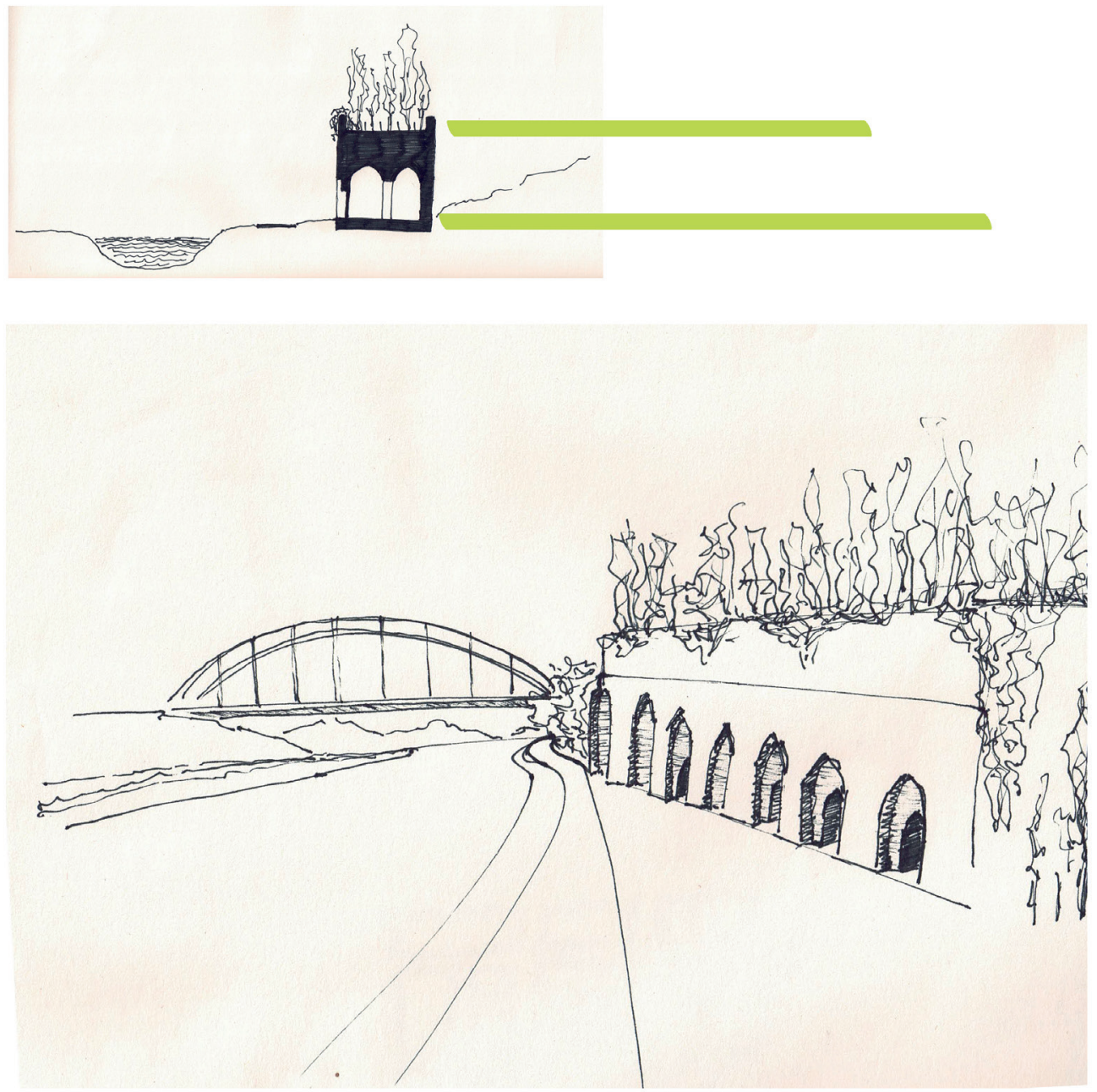

Fig. 6 - Fours à chaux, Tournai, Belgio. Un progetto di layers: tra architettura e natura (elaborazione: G. Vannelli). 

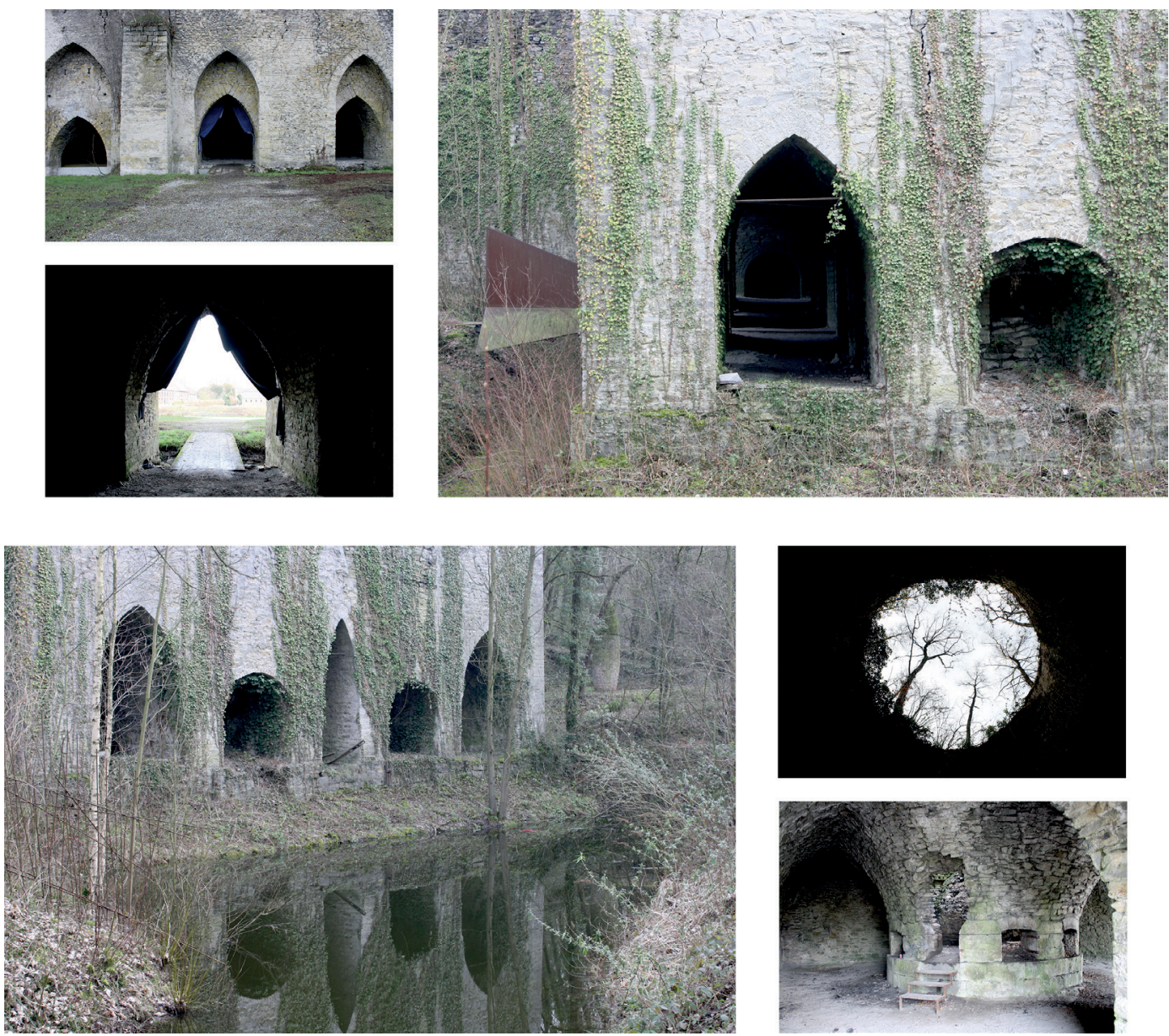

Fig. 7 - Fours à chaux, Tournai, Belgio. Un progetto di layers: il tempo e le tracce (foto: G. Vannelli). 
L'ipertopia - definita da Casetti nell'interrogarsi circa possibili evoluzioni, involuzioni o sparizioni dell'eterotopia del cinema - è qui proposta quale figura retorica del discorso progettuale sulla teoria del re-cycle. Questa trasposizione dal campo semantico della teoria del cinema all'architettura intende tracciare un nuovo possibile orizzonte di senso per le cittadelle cimiteriali che non può esser definito se non mediante una complessa azione progettuale al contempo tangibile e intangibile. Rispetto ad una moltiplicazione ed una contaminazione di tecniche, mezzi e luoghi - condizione valida nella contemporaneità tanto per il cinema quanto per lo spazio cimiteriale - solo la complessità può costituire una plausibile risposta. Un progetto che ha come obiettivo la trasformazione di una eterotopia in una ipertopia - e dunque il passaggio da spazio altro a spazio pubblico - è un progetto che deve tenere il paesaggio al centro (Cortesi, Cappiello, 2017), un progetto che sappia essere relazionale e non statico, un progetto processuale (Bocchi, 2014, p. 19) che continuamente si costruisca nel tempo con azioni di innesco, verifica e modificazioni.

La ricerca che ha ingenerato il progetto R.I.P. - svolta in collaborazione tra Unina e ULiège - ha messo a punto una prima comparazione volta ad approfondire la conoscenza dell'oggetto di studio - il cimitero italiano - mediante l'investigazione di altre tipologie di spazi cimiteriali. In Nord-Europa si possono rintracciare alcune utili lezioni per un progetto di ipertopie cimiteriali. Non a caso, lo spazio per la sepoltura nella tradizione nordeuropea si misura sempre con caratteri e dimensioni del paesaggio travalicando l'idea di cimitero architettonico tipicamente italiana. Mediante tre casi studio si propongono di seguito altrettante possibili strategie progettuali: un progetto di stanze, un progetto di margini e un progetto di layers.

\section{Un progetto di stanze}

Lo spazio claustrale è l'archetipo del camposanto e quindi del cimitero. II chiostro è una stanza a cielo aperto che struttura i complessi sistemi delle architetture conventuali, non troppo dissimili dalle eterotopie foucaultiane. Allorquando il cimitero è un ambito urbano perimetrato da un recinto può essere inteso come una grande stanza urbana, ciò è ancor più evidente se lo si legge in relazione alla più complessa geo-grafia di recinti. II progetto di ulteriori e variegate stanze all'interno del più grande sistema urbano cimiteriale può garantire interessanti intrecci spaziali.

Lo Schoonselhof Cemetery si distende per circa 84 ettari nel distretto di Wilrijk, entrando in contatto con il Fort 7 del National Redoubt belga, strategica cintura difensiva per la città di Antwerpen. Posto dunque ai margini della città, proprio sulla circonferenza tratteggiata dai forti difensivi, Io Schoonselhof si colloca laddove, a sud, la tradizionale morfologia del tessuto residenziale nord europeo cede il passo ad un ampio sistema di magazzini e industrie. Ancora, i sistemi naturali del paesaggio belga si contaminano e si intrecciano con gli insediamenti urbani che, attestandosi lungo le principali vie di accesso, dalla densa città si prolungano verso l'entroterra. Questo è l'attuale contesto di quella tenuta signorile di Schoonselhof che nel 1911 l'Assessore alla Cultura della città belga decise di acquisire come cimitero comunale. II castello con i suoi giardini sono stati per diversi secoli residenza per aristocratici: sin dal 1943 il valore paesaggistico e monumentale del sito ha determinato azioni politiche di tutela.

Questa premessa risulta essere fondamentale al fine di chiarire come questo cimitero sia in effetti l'esito di una storica forma di re-cycle. I caratteri propri del giardino signorile sono divenuti l'assunto di partenza per un progetto in continua evoluzione che nel tempo ha ospitato anche sepolture per musulmani e per ebrei, giardini di dispersione delle ceneri, cinerari e finanche il crematorio stesso. 
II progetto di stanze, insito nella conformazione del giardino di tradizione francese, è divenuto il contemporaneo approccio al progetto di questo sito. A garantire la possibile compresenza di utenze differenti e una percezione serena degli spazi cimiteriali, stanze vegetali danno forma al cimitero-parco utilizzando variamente alte alberature, basse siepi e sistemi di raccolta delle acque. Questo disegno non resta senza seguito: anche gli interventi architettonici realizzati per accogliere i cinerari disegnano spazi conclusi e rimandano a un progetto di stanze. Dunque, lo Schoonselhof Cemetery è un parco degno di nota in cui un attento progetto di architettura e del paesaggio garantisce la compresenza degli spazi dedicati alle sepolture - stanze vegetali o architettoniche - e di spazi pubblici che si configurano come una sistemica concatenazione di luoghi in-between. Questa strategia se da un lato garantisce riservatezza a chi vive il trauerarbeit, dall'altro assicura la definizione di un articolato e variegato sistema di spazi pubblici e attraversamenti che rendono lo spazio cimiteriale un valore aggiunto per la città che ne lambisce i margini.

\section{Un progetto di margini}

II margine è tema quanto mai centrale nel progetto delle eterotopie. Costruire eterotopie significa edificare margini. Costruire margini significa escludere un fuori da un dentro. Eppure, quanto detto si verifica allorquando l'operazione progettuale riguarda ciò che è contenuto o ciò che è escluso, quando il margine diventa perimetro. Se si compie un'operazione di centralizzazione del margine, se si trasla l'inquadratura del foglio ad accogliere il margine nel progetto, a considerarlo parte di un sistema più complesso che non si limiti al margine stesso, allora edificare margini può non significare costruire eterotopie. II margine del Begraafplaats Hoog di Kortrijk diviene l'orizzontale paesaggio belga. II cimitero progettato nel 1995 da Bernardo Secchi e Paola Viganò è un progetto dalle modeste dimensioni ma dal notevole interesse.
La disposizione a terrazzamenti rivolti verso il paesaggio che si lasciano la città di Kortrijk alle spalle interpreta magistralmente, proprio al margine della città stessa, il tema del margine dell'eterotopia. In uso sin dal 2000, questo progetto muta con il passare del tempo e ciclicamente evolve con le stagioni, è un progetto d'architettura fatto con il paesaggio. Costeggiando i terrazzamenti che degradano verso la quota inferiore, un sentiero lastricato immette il visitatore nel paesaggio ricongiungendosi con una rete di percorsi ciclopedonali che si distendono nella direzione ortogonale. Le sepolture trovano spazio sia nei terrazzamenti che nelle strutture verticali disegnate da Secchi per accogliere le urne che, insieme alle alberature delimitanti il percorso, sono i soli elementi verticali del progetto. L'ondulato paesaggio fiammingo diviene un margine vivo e attivo del progetto a partire dalla cresta a nord che definisce la quota su cui si imposta la strada di accesso al sito. Qui l'architettura entra in contatto con la modellazione del suolo. Un progetto, quello di Secchi e Viganò che necessita d'esser letto in sezione, la rappresentazione che meglio di-mostra le relazioni. L'accesso è disegnato da un piazzale parzialmente erboso. Questo spazio pubblico in direzione nordsud media tra la strada e il cimitero. Nella direzione opposta segna invece la distanza tra gli ambienti di servizio - con gli adiacenti impianti sportivi - ed il piano inclinato che dalla quota stradale sale in contropendenza a coprire gli spazi per le cerimonie rivolti verso il cimitero.

A questo paesaggio, nel 2011, si affianca il Crematorium Uitzicht ad opera di Souto de Moura che entra magistralmente in dialogo con il paesaggio fiammingo ma soprattutto con l'interpretazione che precedentemente Secchi e Viganò ne avevano dato attraverso il progetto. II cimitero Hoog-Kortrijk è un sereno luogo di distensione tutto afferente al paesaggio che lo contiene e plasma. Il crematorio si può intendere quale contemporanea interpretazione dell'archetipo del tumulo che, nel relazionarsi con 
il suolo, assume un inedito accento di leggerezza e sospensione: una mastaba scavata e solcata che conforma il margine del paesaggio. La quinta facciata viene esaltata da un progetto attento di un tetto verde che si integra nel contesto naturale con elementi e linguaggi propri dell'architettura. Lo scultoreo camino del forno crematorio svetta a diventare landmark, punto di riferimento per gli spazi pubblici che il cimitero offre alla città.

\section{Un progetto di layers}

Ad una 'città per parti' si affianca, si sostituisce, si riammaglia sempre più frequentemente una 'città per layers'. Da una città letta in pianta si passa ad una città comprensibile solo in sezione. Questa è l'operazione necessaria, da un lato, per reintrodurre nelle dinamiche urbane contemporanee tutti gli strati dei complessi palinsesti su cui viviamo e agiamo con il progetto, dall'altro, al fine di progettare l'intreccio, la contaminazione e quindi la complessità. Ciò è quanto mai vero nel caso delle città altre. A città recintate, affiancate, non dialoganti devono corrispondere nel progetto contemporaneo città sovrapposte e interrelate: i sistemi statici che abbiamo ereditato vanno trasformati in sistemi aperti e dinamici.

Interessante intreccio tra patrimoni, paesaggi e comunità è rappresentato dal singolare caso dei Fours 116 àchaux di Tournai.
In prossimità del centro città, lungo il corso dell'Escaut, le fornaci permangono a rammentare il fervido passato industriale della regione. Dopo un abbandono durato anni, nel 1997, al fine di evitarne la demolizione, questo frammento di archeologia industriale è stato acquistato da privati. Tale condizione è stata premessa proficua per l'avvio di un processo teso a rivalutare questo patrimonio e a determinare una quanto più ampia condivisione del bene. La fondazione FaMaWiWi è da anni impegnata per il riuso dei forni di calce assumendo un approccio sensibile alla costante azione del tempo e alle relazioni che l'ex sito industriale ha intessuto con il paesaggio fluviale su cui si erge. II seducente edificio in pietra, lambito da uno specchio d'acqua in cui si riflettono gli archi ogivali che caratterizzano l'austero prospetto, nel tempo dell'abbandono si è arricchito di un giardino pensile di betulle nate spontaneamente sulla copertura. Questo è divenuto l'occasione per un'interessante sperimentazione progettuale e processuale di valorizzazione che prevede la riconversione dell'antica fornace in un luogo dedicato all'arte, alla memoria e alla comunità. Se da un lato, gli ambienti voltati a piano terra sono utilizzati per eventi pubblici e manifestazioni artistiche facendo rivivere la fornace come luogo di produzione culturale, dall'altro, negli spazi dello spontaneo giardino pensile si pratica la dispersione delle ceneri. 
Inoltre, la copertura delle fornaci diviene gradualmente un parco dedicato all'arte grazie alla prassi dei passe-mémoire: colonnine in ghisa, disseminate tra i tronchi, che supportano piccole opere d'arte finanziate da coloro i quali hanno scelto di far disperdere le proprie ceneri o di lasciare una traccia del proprio passaggio in questo luogo. I Fours à chaux costantemente incrementano il proprio valore identitario: il significativo patrimonio industriale sottratto alla demolizione è divenuto, da un lato, luogo di aggregazione per la collettività e, dall'altro, eterna dimora per coloro i quali hanno animato in vita quegli spazi. Inoltre, un ulteriore spazio pubblico è proposto lungo il fiume: il 'giardino delle nascite'. Nel ricordare le nascite annuali piantumando nuovi alberi, questo spazio è proposto quale ulteriore segno di una rinascita complessiva.

\section{Verso l'ipertopia}

Così, si propongono una percezione e un ruolo inediti dello spazio di sepoltura: non più deposito di memorie bensì spazio destinato ad una comunità che ritrova nell'ipertopia cimiteriale un luogo di incontro, nell'arte e nella cultura strumenti di rigenerazione e nella memoria un valore collettivo.

Le strategie descritte sono qui proposte come possibili presupposti e premesse per il progetto di uno spazio cimiteriale ipertopico, laddove l'azione pro- gettuale lavora con l'architettura e il paesaggio a conformare i lastscapes come luoghi catalizzatori di spazi, come punti di accumulazione di comunità e significati, come paesaggi disponibili per mutevoli geografie della publicness, spazi pubblici inclusivi e complessi rispondenti ad una visione multifunzionale del paesaggio.

Quando il progetto dello spazio e dell'architettura opera uno slittamento concettuale teso ad accogliere la dimensione del paesaggio nel suo essere relazionale, mutevole e dinamico, le stanze divengono la traccia al negativo di una geografia dell'in-between, il margine non si conforma quale recinto ma diviene soglia, i layers danno forma alla compresenza e all'intreccio. Lo spazio altro diviene spazio pubblico e l'eterotopia trova le premesse per essere ipertopia in cui il 'qui' si scioglie 'nell'altrove'. 


\section{Note}

1 II paragrafo Una geo-grafia di recinti: tra spazio pubblico e publicness è attribuibile a Rita Occhiuto, il paragrafo Lastscapes: obsolescenza tra memorie e nuove istanze ad Angela D'Agostino, il paragrafo Verso l'ipertopia: tre strategie progettuali a Giovangiuseppe Vannelli.

${ }^{2}$ Rethinking lastscape Perspectives (R.I.P.) è un progetto di ricerca finanziato tramite un Bando per il Finanziamento della Ricerca di Ateneo dell'Università degli Studi di Napoli Federico II. II P.I. è la prof. A. D'Agostino mentre G. Vannelli è membro del gruppo di ricerca. La ricerca si avvale della pluriennale collaborazione con la prof. R. Occhiuto (Université de Liège). 


\section{Bibliografia}

Abramson D. M. 2016, Obsolescence: An Architectural History, The University of Chicago Press, Chicago.

Barberan F.J.R., La memoria abitata. Gli spazi della morte nella cultura europea contemporanea, in Felicori M. (a cura di), Gli spazi della memoria. Architettura dei cimiteri monumentali europei, Luca Sossella Editore, Roma.

Bocchi R. 2014, Appunti su bricolage, riciclo, merz-baue pratiche urbane dal basso, in Paolella A. (a cura di), People Meet in the Re-cycled city, Aracne editrice, Roma. pp. 17-20.

Bocchi R. 2017, Progetto di nuovi cicli di vita per i territori italiani del XXI secolo, in Fabian L., Munarin S. (a cura di), Re-Cycle Italy. Atlante, LetteraVentidue, Siracusa. pp. 7-10. Casetti F. 2015, La Galassia Lumière: sette parole chiave per il cinema che viene, Bompiani, Milano.

Cerri P., Giovannoli R., Neeff F. 1979, Recinti, «Rassegna», n. 1, pp. 7-32.

Cortesi I., Cappiello V. 2017, II paesaggio al centro. Integrazione tra discipline, LetteraVentidue, Siracusa.

De Leo E. 2006, Paesaggi cimiteriali europei. Lastscape realtà e tendenze, Mancosu editore, Roma.

Foucault M. 2011, Spazi altri, in Vaccaro S. (a cura di), Spazi altri. I luoghi delle eterotopie, Mimesis Edizioni, Udine.

Gregotti V. 1979, Editoriale, «Rassegna», n. 1, pp. 5-7.

Heathcote E. 1999, Monument builders, Academy Editions, Londra.

Mangone F. 2004, II sistema cimiteriale napoletano, tra storia e attualità, in Mangone F. (a cura di), Cimiteri Napoletani. Storia, arte e cultura, Massa Editore, Napoli.

Masullo A. 2004, L'eloquente artificio della memoria e la silenziosa emozione del ricordo, in Mangone F. (a cura di), Cimiteri Napoletani. Storia, arte e cultura, Massa Editore, Napoli.
Morelli U. 2020, I paesaggi della nostra vita, SilvanaEditoriale, Cinisello Balsamo (MI).

Morin E. 2000, La testa ben fatta. Riforma dell'insegnamento e riforma del pensiero, Raffaello Cortina Editore, Milano.

Venturi Ferraiolo M. 2001, "Trasformare in giardino il mondo": Rosario Assunto e l'etica della contemplazione, in Luciani D. (a cura di), Luoghi. Forma e vita di giardini e paesaggi, Edizioni Fondazione Benetton Studi Ricerche/ Canova, Treviso. 